\title{
Experiences in Alzheimer's Disease: What Do Stakeholders Post on the Internet?
}

\author{
Lauren S. Seifert, Kara Kaelber, Kathleen Flaherty, \& Tyler J. Bowman \\ Malone University, Canton, Ohio, USA
}

\begin{abstract}
Alzheimer's disease $(A D)$ is a neurodegenerative condition that is chronic, progressive and terminal across an interval of approximately five to ten years from the time of diagnosis. Disease management in $A D$ and other illnesses has been conceptualized in a number of ways, including the Health Co-Inquiry approach (a cooperative endeavor that activates stakeholders, regards all stakeholder views, uses evidence-based practice, and strives for integrated care). With growth of Internet use, Health Co-Inquiry may occur online as stakeholders in chronic disease management search for and give information and support. The current study is a first look at the online posts of all AD stakeholders. Our goal is to shed light on their experiences by characterizing the themes in their posts. A Bifurcated Method was used with quantitization of information through a web-crawling program in order to corroborate or refute the findings from an inductive, thematic (qualitative) analysis. Results indicate that $A D$ stakeholders utilize the Internet for: information, assistance, emotional support or relief, and advice about decision-making and behaviour challenges. Persons with AD posted little compared to caregivers and were prone to report subjective cognitive impairment (SCI) and ask for help regarding diagnosis. Caregivers were apt to divulge personal stories, ask whether they should deceive a person with $A D$, and request information about coping or note that posting online was a coping mechanism. Providers', agencies' and companies' posts were likely to give information and offer technical advice, answer specific caregiver questions, and offer paid services/clinical trials. As a first study of all AD stakeholder online narratives, data indicate that more than half of sites and posts are dedicated to the concerns of caregivers and persons with $A D$, indicating that they are engaging in some aspects of Health Co-Inquiry online.
\end{abstract}

Keywords: Alzheimer's disease; Health-Co-Inquiry; Internet research; blog; forum

\section{Introduction}

What do stakeholders in Alzheimer's disease (AD) communicate online? Do they use the Internet in disease management? If so, what functions might their posts serve? The purpose of this study was to explore online posts by stakeholders and discern key themes in their narratives, thereby filling a gap in published research about AD.

Because our goal was to uncover the specific disease management (and, perhaps, other) purposes of $A D$ stakeholder posts by way of the themes expressed in them, we adopted the Bifurcated Method of Seifert et al. (2019). It utilizes qualitative, thematic, inductive analysis along with quantitization of themes via a computer program. Qualitative analyses enable researchers to identify and explore the content of narratives, while quantitization of themes serves as a cross-check and bolsters rigour.

In addition, our study was conducted from a perspective emphasizing Health Co-Inquiry (Seifert \& Seifert, 2017), which highlights stakeholder cooperation in chronic disease management. At its best, Health Co-Inquiry includes 
person-centered care, stakeholder activation, evidence-based practice, and if possible, integrated care through collaborative processes to manage chronic health and mental health conditions (Seifert \& Seifert, 2017). When we began this project, in addition to the queries posed above we wondered, "Do Internet posts of stakeholders in AD show signs of Health Co-Inquiry online?" We share more about the rationale for our project, the Bifurcated Method, and Health Co-Inquiry in upcoming paragraphs.

\section{Why Should We Study AD? The Global Challenge}

Alzheimer's disease (AD) is a progressive, neurodegenerative condition for which there is no known cure. As people live longer, healthier lives, this neuropathology has reached epidemic proportions in the aging population, with an estimation that the number of those affected may double in each twenty-year interval between 2010 and 2050 from 36 million to nearly 115 million (Prince et al., 2013). In the UK, persons living with dementia exceeded 850,000 in 2015, and the number is expected to be approximately 2 million by 2050 (Alzheimer's Research UK, 2018). According to the Xu et al. (2016), in the United States AD is among the top ten causes of death. The epidemic of $A D$ is not only a national problem; it is a worldwide catastrophe (Lozano et al., 2012).

\section{What Types of Support Help AD Stakeholders?}

Contextual supports (like sensory, environmental, and social cues) are vital in AD management (Kenigsberg et al., 2016; Woodbridge et al., 2018), and so are pharmaco-therapeutic interventions for cognitive and behavioural symptoms (such a cholinesterase inhibitors and memantine; van de Glind, 2013). Social support is beneficial for persons with AD and other disease stakeholders (Bassuk et al., 1999; Han et al., 2016; Krueger et al., 2009; Lum \& Lightfoot, 2005; Williams \& Kemper, 2010). Furthermore, Whitlatch and Orsulic-Jeras (2018) identified the following vital buttresses in AD: providing information about the disease and its progression, counseling and emotional support, help with everyday tasks and activities, respite and time away from caregiving, multi-dimensional programs that help with two or more of the above and/or help people access leisure, medical care, and/or financial assistance. Presumably, the Internet may be used to locate any or all of the above, and interpersonal cooperation is increasingly happening online (Stewart, 2013).

\section{What Types of Support Help Stakeholders in All Chronic Diseases?}

One's social connections are important predictors of short- and long-term outcomes in chronic illness (HoltLunstad et al., 2017), and interpersonal interactions (whether online or offline) may be essential for gathering information and garnering emotional and physical support (Frerichs et al., 2016; Pietromonaco \& Collins, 2017). In particular, when a person has access to such support, it can mitigate role strain and increase role affirmations (as with caregivers who learn that others are going through similar experiences; Goode, 1960; Marks, 1977; see also, Cohen \& Wills, 1985; Luoh \& Herzog, 2002; Oshio \& Kan, 2016; Stanhope \& Henwood, 2014; Sugihara et al., 2008). On the other hand, social disconnections (e.g., hostility, isolation, alienation) may interfere with disease management (Pietromonaco \& Collins, 2017).

\section{Disease Management and Growth of Internet Use}

A new Technology Era began around the recent turn of the century, with daily use of the Internet by large portions of the general population. Since then, daily online user activity has expanded to more than 4 billion from just 500,000 (Internet Live Stats, 2018). Research on chronic illness points to Internet use for information seeking and social support (Ahola Kohut et al., 2017). The characteristics of one's personal, offline support in chronic disease management help predict greater use of online support and may be an outgrowth from the former, and it is possible that persons who are less happy seek more online support (Allen et al., 2019). Social networks, both online and off are vital for the promotion of self-care and improved day-to-day outcomes in chronic illness, including AD (see Rogers et al., 2011; Vassilev et al., 2011). 


\section{Older Users, Dementia, and Internet Support for Disease Management}

With respect to older adults online, research indicates that a tailor-made computer system (with vetted Internet links and resources, a photo tool, a calendar, games, internet assistance, and email) enhanced their perceptions of social support and well-being, while reducing loneliness over six months as compared to those who received a hard copy of a binder with some similar resources (Czaja et al., 2018). For persons with dementia (PwD) technical support seems to enhance use of computers for things like playing games and email, although the relative amounts of online engagement time seem to be much lower than for older adults without dementia (Tak et al., 2015; LaMonica et al., 2017). For informal caregivers of persons with dementia, research about computer use points to scheduling ("Calendar") features and a resource for sharing photos and narratives (i.e., "Timeline") as the most beneficial parts of a multi-faceted social media platform (called "Inlife"; Dam et al., 2017). Among the advantages, Dam et al. (2017) reported improved coordination of care for persons with dementia. Furthermore, Scharett et al. (2017, p. 643) noted the well-being of caregivers as the most frequent "solution" (via responses to posts) on a single, online caregiver platform.

\section{Using the Internet as a Cooperative Mechanism for AD and/or Other Disease Management}

Given the foregoing, it makes sense to assert that stakeholders in AD and other chronic diseases might use the Internet as a mechanism for gathering information, giving information, sharing resources, and receiving or providing other types of support. Online interactions and resources may become part of care management and self-care for persons with AD and related dementias, as in the case of the InLife Timeline feature, which can be used to help cue a person with dementia with photos in order to remind her or him about events, people, and social exchanges (Dam et al., 2017). Such online mechanisms could become keys in a collaborative endeavor whereby stakeholders increase their activation toward better management and outcomes while respecting each other's views, working toward evidence-based practices, and striving for integrated care of the whole person. If those things happen-whether online, in person, or in some combination of the two-then Health Co-Inquiry is occurring (Seifert \& Seifert, 2017).

Identified as a collaborative approach to the management of chronic conditions, Health Co-Inquiry may include numerous features. Among them, Seifert and Seifert (2017) recommended person-centredness and activation, whereby all stakeholders' perceptions and wishes are regarded and there is promotion of all stakeholders as engaged participants in disease management. Furthermore, Seifert and Seifert advocated evidence-based practice and integrated care so that treatment decisions are guided by sound research and coordinated across specialties to accomplish whole-person care. The relevance of Health Co-Inquiry to online posts by stakeholders in AD is that the acts of posting may be evidence of stakeholder activation and collaboration-seeking intentions. The current study may be a first step in understanding how online experience could be part of Health Co-Inquiry for stakeholders in $A D$ and related dementias. One goal was to discern themes (using the Bifurcated Method; Seifert et al., 2019) in order to establish whether AD stakeholders are taking part in Health Co-Inquiry online.

\section{Cooperative Engagement and "IIIness Work"}

Because we utilize the Health Co-Inquiry approach we are especially interested in the cooperative and socialsupporting functions of online posts among stakeholders in AD and other chronic illnesses. In our view, both online and offline behaviors may be part of Health Co-Inquiry (i.e., activating stakeholders, regarding all stakeholder views, using evidence-based practice, and striving for integrated care; Seifert et al., 2019). As was noted above, as a chronic disease, AD presents some of the same challenges as other chronic conditions (e.g., self management, management of the condition, and case management), although individual persons and illnesses have unique characteristics (Rogers et al., 2011). So, it is logical to extend findings about support in the management of chronic conditions to AD, as well (Marks, 1977).

In a seminal article, Corbin and Strauss (1985, p. 224) described three essential types of "work" that are part of daily management of chronic conditions ("illness work"): (1) tasks linked to disease management, (2) activities related to everyday activities, and (3) "biographical work". Rogers et al. (2011) further conceptualized illness work in five classes, with some duties regarding restoring normalcy, others bringing new information into practical applications, a third set related to how things get done, a fourth for determining who can do things, and a fifth 
constellation that is emotional (e.g., providing comfort and reassurance). All of those tasks are part of the management of $A D$, and some illness work can happen online. Furthermore, those online activities may cut across illness work categories (e.g., communicating with caregivers and health providers, coordinating prescriptions/treatments, finding and offering emotional support, and gathering information).

\section{The Current Study}

Given the foregoing, it is reasonable to assert that online posts are an important part of the growing online world for the general public. Furthermore, we posit that resources, information, and social support on the Internet can be a vital part of Health Co-Inquiry whereby stakeholders seek and provide information and support online. As such, online activities become part of Health Co-Inquiry, because they constitute one or more aspects of it: being activated in ways related to disease management and personhood, being motivated toward integrating aspects of care, and or being oriented toward information and support seeking/giving.

For researchers, online posts may be utilized as a source of data, as an indicator of the needs, wants, and behaviours of people with $A D$ and related types of dementia, their caregivers, their health and mental health providers, and various agencies/organizations involved in AD research and care. How, though, is stakeholders' use of the Internet for disease management best studied? Some researchers have collected large amounts of Internet data and quantitized themes by counting specific types of posts (Kim et al., 2016; Scharett et al., 2017). Others have relied on inductive analyses in order to discover key themes (Coulson \& Greenwood, 2011; Paulus \& Varga, 2015). We did both, adopting the Bifurcated Method (Seifert et al., 2019) so that we might discern key themes in AD stakeholder posts and add rigour to our analyses with quantitization of themes using a web-crawling computer program. Our approach is consistent with Greene's (2007) argument that the two techniques (i.e., inductive analysis and quantitization of themes) can complement each other. It is our belief that the Bifurcated Method provides a fitting means by which to investigate our research questions:

- What do stakeholders in Alzheimer's disease (AD) communicate online?

- Do they use the Internet in disease management?

- If so, what functions might their posts serve?

- Do Internet posts of stakeholders in AD show signs of Health Co-Inquiry online?

\section{Method}

As noted, we utilized the Bifurcated Method that originated with Seifert (2015) and which has been further delineated by Seifert et al. (2019). As part of this method, we conducted thematic analyses of posts on Internet sites related to AD, and we used a program to "crawl" those URLs ("Uniform Resource Locators", also called "web addresses"). While it crawls the Internet, counting the frequencies of key terms that we specify, the researchers conduct independent inductive, thematic (qualitative) analyses of the narratives posted by AD stakeholders at the URLS.

Table 1 shows the steps of the Bifurcated Method as we applied it to the study of AD. The Bifurcated Method can be applied to just about any topic for which there are posts online, and Seifert et al. (2019) offer assistance to other researchers who use the approach. Essentially, the technique utilizes inductive, thematic (qualitative) analysis (Hatch, 2002) and a "web crawl" using a robot program. The former is conducted by the researchers, who use four search engines (Google ${ }^{\mathrm{TM}}$, Yahoo ${ }^{\mathrm{TM}}$, $\mathrm{Ask}^{\mathrm{TM}}$, and Bing ${ }^{\mathrm{TM}}$ ) to explore the Internet for forums, bulletin boards, and websites that are dedicated to a topic, like AD and related dementias. All URLs are then vetted to be sure that they are pertinent, and those web addresses are given to a robot program so that it can search them for keywords (see Appendix A; with the initial, unvetted URL list available from the authors). The web crawl is accomplished via a dedicated program, which uses a variety of different types of code (e.g., Java, HTML, CSS, JavaScript). The program was written by a software designer and was made possible through a grant for technological development in research to our institution. 
Step 1: Identify Alzheimer's Disease (AD) as a chronic condition for scrutiny with respect to stakeholder narratives online.

Step 2: Use the global dictionary of health terms created by Seifert et al. (2019) and develop a condition-specific dictionary of search terms for AD.

Step 3: Use search engines to find websites with posts by stakeholders in AD.

Step 4: Vet the websites from Step 3 using inclusion and exclusion criteria.*

Step 5: Each researcher evaluates content and discerns themes at 10 randomly selected websites from the Step 4 list. **

Step 6: Researchers meet to discuss and compare their reflections. This leads to compilation of a list of key themes found in stakeholder narratives online.**
Step 5: Identify a web-crawling robot program that can be used to collect stakeholder narratives online.

Step 6: Use the web-crawling robot program to search for all terms in the global and condition-specific dictionaries. Also, use the program to discern frequencies of dictionary terms at each website.

Step 7: Data from the web-crawling robot can be used to help instill rigour in the researchers' inductive, thematic analysis. Researchers evaluate consistency between key themes from the researchers' inductive, thematic analysis and prevailing themes from the web-crawl word frequency data.

Step 8: An additional step might be needed if Step 7 yields inconsistencies. If so, the researchers must carefully scrutinize the key themes from their inductive, thematic analysis and themes from the web-crawling robot program.

Notes. *Criteria are described in the current manuscript. Methods are adapted from those of Seifert et al. (2019).

**Steps 5 \& 6 of the thematic analysis (on the left) are completed without consulting the web-crawl results.

A dictionary of keywords is created and loaded into the robot program by the researchers, who search their university's online databases for the ten most recent books on the topic of interest, taking terms from the Table of Contents of each book and removing duplicate terms. Respectively, Appendix A names the URLs that were vetted and searched by our robot program. Appendices B and C provide a condition specific dictionary of terms and a global dictionary related to chronic health conditions; they were supplied by Seifert et al. (2019).

Websites were accessed for data analyses from 12 June 2017 to 6 January 2020. Dates of posts on the URLs were between September 2007 and November 2017. Two sites had initial copyright dates earlier than those dates: one as 1996-2019 and another as 2003-2019. Rather than being forums, the latter two websites were associated with an informational site for health and mental health providers and a directory of URLS related to AD and related dementias, respectively; the two sites did not permit posts by other users.

\section{Inductive, Thematic (Qualitative) Analyses}

Using the method of Hatch (2002), we first decided on frames of analysis (i.e., the basic units of narrative that we would study). We established that our frames of analyses would be individual posts and then decided that our domains (ways of grouping the frames) would be stakeholder groups. Furthermore, we identified key groups for further study, persons with $A D$, caregivers of persons with $A D$, mental health and health providers, and agencies/organizations involved in research and/or care for persons with AD. For the purposes of this study, providers and agencies were grouped as a single domain. Each of the four researchers was provided with a list of ten URLs selected at random from among the 80 websites we had gathered (with 20 from each search engine). The ten websites for further qualitative analyses were selected at random from among those URLs that were being crawled by the robot program.

In our thematic analysis, each researcher visited the "random ten URLs" on her or his own and completed a spreadsheet while reading information on the web-pages and in people's posts. It is critically important that researchers did not converse with each other about the themes during the interval when they were independently working to devise their own lists of themes.

Each researcher responded to the following:

- What is the main purpose of the URL?

- What appears to be the purpose of the particular posts/passages you have read? 
- What are your insights about this URL and its narrative(s)?

- Provide at least one quote that is consistent with your insights.

After completing their spreadsheets, all researchers' files were sent to the first two authors for compilation and comparison. Then, the researchers met three times to discuss the themes they had discerned (and without reading the other researchers' pages). All researchers met at the initial "themes meeting"; authors one, two, and four attended the second session. Finally, the third session involved only the first and second authors, who compiled the list of all themes which had been discussed and discerned in the previous meetings. Only then, did the researchers consult the web-crawl (quantitized) results for comparison with their inductive, thematic data. Our threshold for inclusion of a theme in the results of our qualitative analyses was that three of four researchers (75\%) must have identified it in their independent analysis of narratives and have agreed to have the theme included in our final list of qualitative results.

\section{Quantitization of Keywords at URLs}

The web-crawling program provides the second prong of the Bifurcated Method (on the right in Table 1). Once the URLS and dictionary of keywords were loaded into the robot program, it counted the frequencies of the terms at each website. This provided a "quantitizing" approach to analyzing the narratives. Before the web crawl, we categorized the keywords in our dictionary (with respect to their domains and topics; e.g., "Alzheimer" as a general term that might indicate any number of themes or behaviors, "mother" and other family terms as markers of caregiver concerns, "treatment" as a term that usually suggests information seeking or giving). See Table 3 for a list with the domain/theme categories.

After duplications had been removed from our original list of 80 websites (from search engines, as mentioned above), 70 viable URLs remained, but 36 of them could not be searched by a robot program. The 36 "uncrawlable" websites were eliminated from additional consideration; we did not use additional programs for analyses (such as NVivo ${ }^{\mathrm{TM}}$ ) because we were already using two techniques for analysis-one of which is a dedicated web-crawling program. From among the 34 URLs that remained, all were "crawled" by our robot program in order to establish word counts, and this is described in further detail below.

Table 2. A Comparison of Types of URLs Among the Crawled and Uncrawled Sites.

\begin{tabular}{lcc}
\hline Type of Site & Crawled URLs (34) & $\begin{array}{c}\text { Uncrawled URLs (36 - 3 } \\
\text { sites taken down: 8.2\%) }\end{array}$ \\
\hline Forum for anyone/diverse topics (not necessarily specific to AD) & $8.8 \%$ & $2.8 \%$ \\
Forum for patients and caregivers & $41.2 \%$ & $27.8 \%$ \\
Forum for caregivers only & $11.8 \%$ & $38.9 \%$ \\
Forum for patients only & $5.9 \%$ & $0 \%$ \\
Focus: Commercial product or advertisement for AD stakeholders & $5.9 \%$ & $8.3 \%$ \\
Directory of URLs & $2.9 \%$ & $0 \%$ \\
Informational site for professionals/researchers & $5.9 \%$ & $0 \%$ \\
Informational site for any AD stakeholder or & & $2.8 \%$ \\
Expert advice for patients and caregivers (i.e., usually with an “Ask & $8.8 \%$ & $2.8 \%$ \\
Dr. X" or chat format) & $2.9 \%$ & $5.6 \%$ \\
Health service/Government agency/Non-profit agency website & $5.9 \%$ & $2.8 \%$ \\
Media outlet & $0 \%$ & \\
Site about another topic on which conversation about & & \\
AD/dementia is taking place & & \\
\hline
\end{tabular}

Of the uncrawlable websites, all were reviewed by the first author in order to determine whether those URLs had markedly different content than the ones that were crawled. The identifiable difference was that the crawled URLS permit programs to count their words, while the uncrawled sites do not (e.g., through blocking, by requiring a subscription). Table 2 provides a comparison of the types of sites in the lists of crawled and uncrawled URLs. They 
are similar, with the most populous sites being forums for patients and caregivers or for caregivers only and with the distributions of sites being somewhat similar, although the frequencies of specific types of sites do differ across the two lists.

At the end of our method (see Table 1, Steps $7 \& 8$ ), we were able to use the web-crawl results to cross-check the categories and frequencies of the terms against the themes we had compiled in our qualitative analyses. In our method, this is accomplished when themes identified in our qualitative work are compared with the robot program's dictionary word "counts" and their pre-designated categories. This helps to establish whether the themes we have identified in our qualitative analysis are evident in the robot program's word counts. It also helps to indicate the relative prevalence of stakeholder Internet presence (e.g., more caregivers than persons with AD). Moreover, we believe that our word frequency data are useful in ways consistent with Maxwell's (2010) assertions about adding rigour to qualitative analyses through the use of quantitization. When we categorize dictionary terms, run the robot program, and look at word counts (i.e., after our thematic analyses), we are forced to go back to our qualitative analyses and check whether the web-crawl word counts support our qualitative findings.

\section{Techniques for Safeguarding "Subjects": Ethics in Research About Online Artifacts}

Online artifacts are readily available to the general population on websites that do not require passwords for access. As such, using those posts and sites as "data" is consistent with the "exemption" category of review for human research in which there is no contact with humans for the purposes of collecting information (e.g., Office for Human Research Protections, 2018), and the data are publicly available online. Nevertheless, our fundamental premise is that the asynchronous communications afforded by the Internet can provide information and a sense of connectedness for users (e.g., Rubenstein, 2015). As was mentioned above, we wanted to be sure to be respectful of the rights of stakeholders who might have posted at the URLs we studied. In order to help safeguard those persons, our research was reviewed by our university's institutional review board (IRB) and approved before the study began. Among the safeguards we included were: never providing a moniker or user name for a post we quoted in our reports about this project; never posting on any of the sites from which we derived information (which might have created a dual-relationship with the users); and using only those sites that are available without passwords. Random numbers were assigned to all cases, so that we might quote them without users' monikers, and we limited the number of words in sequence that we quoted in this report in order to diminish the chances that a reader might search the Internet and find specific subjects. We are very concerned about the use of online posts as data and urge all researchers who harvest such posts as data to very carefully consider how they use them and what steps they should take to safeguard users (Ngwenya \& Mills, 2014). Also, we welcome readers' suggestions about additional safeguards that might be warranted.

\section{Results}

Here we describe the two types of analyses that we conducted. First, we describe qualitative (inductive, thematic) results. Then, we detail quantitized data from the web-crawling program.

\section{Qualitative Results: Themes Within Each Stakeholder Domain}

Themes were discerned across three fundamental domains, i.e., stakeholder groups representing (1) persons with suspected Alzheimer's or a related disease, (2) caregivers for someone with AD or a related dementia; (3) providers and provider services/organizations. The saturation threshold for reporting a theme is $75 \%$ (3/4), i.e., the theme had to be noted by at least three of four independent raters in order to be documented here.

As examples of stakeholder narratives, we offer quotes from among our URLs. However, we have assigned random case numbers to them (without naming the specific URL where each posted, per our approved IRB protocol). Our goal is not to provide the reader with a road map to find a specific post and its user but, instead, to simply illustrate those themes that we have identified. Persons with AD or suspected AD are called by the moniker "First-Person Case". Caregivers are so-named, and mental health and health providers/organizations are given the label "Provider". Each case also carries a number that is randomly assigned, e.g., First-Person Case 1. As an additional safeguard, in order to make user quotes less "searchable" and individuals less identifiable online, we 
have reduced the number of continuous words in sequences that we have quoted, using ellipses liberally and having tested the quotes in our report by searching Google ${ }^{T M}$ for them (Ngwenya \& Mills, 2014).

\section{First-Person Narratives}

Persons with Alzheimer's disease posted relatively rarely; Table 3 indicates that sites dedicated to their posts were only 5.9\% of all crawled URLs. Quantitization of total number of posts across all author's comments revealed that caregiver posts out-numbered posts by persons with Alzheimer's or suspected Alzheimer's by 10 to 1 up to 20 to 1 , depending on the site. On only one site was the ratio less than that ( 6 to 1 ). In messages by those with AD or a related condition, seeking information was a fundamental theme. Some persons were looking for information about suspected $A D$ and how they might be evaluated or find a health provider who specializes in $A D$. An illustration is a person who asked whether others had or, "...have problems with the...testing?" It seems the user could not complete an online diagnostic test, because of browser incompatibility (First-Person Case 1).

\section{Caregiver Narratives}

Posts by caregivers are by far the most populous on the sites we visited (Table 2). Some message boards are specifically for spouse caregivers, while others include primary and casual (informal) carers. The list of themes for caregivers was the longest of the lists for the three stakeholder domains. Caregivers: (1) seek information, (2) look for assistance (e.g., making decisions, dealing with family dynamics), (3) vent about their emotions, (4) seek emotional support and validation, (5) identify posting on a forum, specifically, as a coping tool, (6) ask, in particular, about whether they should lie to their loved one with AD/dementia, (7) tell their personal stories, and (8) ask for some sort of algorithm or set of rules for how to cope. However, caregivers give information and support, too. Thus, those are important ninth and tenth themes.

Caregiver 7 wrote that an online website devoted to AD, "...keeps me sane....". The user went on to note that although blogging was unusual for her or him, it was part of "...learning..." with a certainty that other people, "...are doing the same." The passage illustrates posting as a coping technique and tool for emotion regulation. It also indicates that the person who posted is using the forum in order to glean information and/or skills (as in the reference to "learning"). Another quote exemplifies the themes of telling one's story and seeking help (especially with family dynamics). Caregiver 6 wrote about her mom that she had branded her primary caregiver as, "...evil and she wants her out of the house..." continuing with an appeal for advice about how to improve the situation. Another carer told her story while giving helpful information to others. She remarked that she would, "...take my Dad for walks" in order to help him stay physically fit. She continued by mentioning that this also helped her to have "...peace." Further, she identified the value of sharing "...a little escape" with him via their mutual enjoyment of music (Caregiver 10).

As an example of why people try to find information on the Internet, we quote from Caregiver 11 who was instructed by a health provider, "...to look online" after having "...no counseling" and very little assistance from "...social services". To respond to such a person, there are both caregiver posts and those by providers. For instance, the "abcdirectory.com" has an Alzheimer's sub-directory that re-directs users to sites with provider resources. In addition, caregivers often respond to other caregivers' calls for help. Caregiver 4 gave, "...research about music...development and the brain...." The user proceeded to quote a Johns Hopkins researcher and then to cite some work about medications for $A D$ and individual differences in patient responses.

\section{Third-Person Narratives}

Provider/agency sites are generally different than message boards for persons with AD and their caregivers. Overall, as one would expect, provider websites are commercial ventures. Providers tend to post fact-based information, like (1) summaries of research results and publications, (2) lists of resources and facts, (3) advertisements for paid services and how to access them, (4) calls for research subjects, and (5) forums for professionals to answer patient/caregiver questions. As an example, Provider 1 posted that readers should look to her/his website as a, "...haven". The URL is hosted by a company for in-home senior care. Provider 2 not only gave summaries of research, job postings, and available grants, but posted an exhibit hall where companies might 
hock their products; the page seemed to be directed at AD providers and researchers. There are numerous similar examples among the pages we evaluated.

Table 3. Highest Frequency Keywords (via the Web-Crawler) and Their Domain or Thematic Categories.

\begin{tabular}{|c|c|}
\hline Keyword Frequency* & Domain and/or Thematic Category \\
\hline Alzheimer (539) & General; all stakeholders \\
\hline III (431) & $\begin{array}{l}\text { General; all stakeholders; } \\
\text { Also, Patient and Caregiver stories; also, part of another word like "will" }\end{array}$ \\
\hline Son (243) & Caregiver-patient relationship \\
\hline Disease (175) & Patient and caregiver stories; General; all stakeholders \\
\hline Dementia (171) & Patient and caregiver stories; General; all stakeholders \\
\hline Work (144) & $\begin{array}{l}\text { Unspecified; may relate to "employment" or to "what works" (colloquial) with regard to } \\
\text { treatments, etc. }\end{array}$ \\
\hline Mother (130) & Caregiver-patient relationship \\
\hline Caregiver (123) & Caregiver-patient relationship; also, paid caregiver employment \\
\hline Husband (121) & Caregiver-patient relationship \\
\hline Good (113) & General; all stakeholders \\
\hline Stage (79) & Diagnosis; also, General; all stakeholders \\
\hline Friend (76) & Caregiver-patient relationship \\
\hline **Best (75) & General; all stakeholders \\
\hline **Sign (75) & Diagnosis; also, General; all stakeholders \\
\hline Doctor (68) & General; all stakeholders \\
\hline Suffer (64) & Patient and Caregiver stories \\
\hline Wife (62) & Caregiver-patient relationship \\
\hline Medical (58) & General; all stakeholders \\
\hline Exercise (55) & Treatments; also, Patient and caregiver stories; Provider/Expert advice \\
\hline Symptom (53) & Diagnosis; Treatment; also, General; all stakeholders \\
\hline
\end{tabular}

\section{Quantitized Results From the Web-Crawling Robot Program}

As we mentioned in our description of the Bifurcated Method (Table 1), near the close of a study, we compare the themes that we have discerned through our inductive, qualitative analysis with the frequencies of theme-relevant terms at the URLs. We use a web-crawling robot program to search each URL for terms that we have specified (i.e., using "dictionaries" as described above). Then we compare the themes represented by the most frequent terms at the URLS to our inductively discerned themes (as with the quotes from Caregivers 7 and 11, above). Table 3 shows the most frequent terms from the web-crawl and names theme-based categories of each one.

Not surprising is the most populous word at the URLs: Alzheimer. It appeared 539 times across the 34 websites (with the total number of words across the URLS $=108,359$ ). In addition, the robot program yielded the following as the nineteen most populous terms after "Alzheimer" (with their frequencies in parentheses): ill (431). son (243), disease (175), dementia (171), work (144), mother (130), caregiver (123), husband (121), good (113), stage (79), friend (76), best (75), sign (75), doctor (68), suffer (64), wife (62), medical (58), exercise, (55), symptom (53). Referring back to the induced themes from our qualitative analyses, we were not surprised about the frequent mentions of family members. When caregivers relate their stories, ask for advice, or provide support, they often mention their relationship to a person with $A D$ (as in the quotes from Caregivers 6 and 10, above). That posters named signs, symptoms, being ill, suffering, disease, nouns that are modified by the adjective "medical", and the term "doctor" was not astounding to us, either, because telling one's story, asking for help, or providing aid are commonly accompanied by mention of the physical and mental states of the person with AD along with statements about his/her caregivers and medical providers. 


\section{Discussion}

Overall, we believe that our findings are consistent with offline research indicating that some AD stakeholders (e.g., persons with $A D$ and their caregivers) may benefit from social support (Bassuk et al., 1999; Han et al., 2016). In addition, our thematic analysis suggests that $A D$ stakeholders can and do use the Internet to connect with others, share information and resources, and give/get emotional support (Frerichs et al., 2016; Pietromonaco \& Collins, 2017). Those themes provide evidence of illness work (Corbin \& Strauss, 1985; Rogers et al., 2011). In turn, illness work online with communication and collaboration within and across AD stakeholder groups suggests Health Co-Inquiry (Seifert, 2015; Seifert \& Seifert, 2017).

We hope that the current study is a first step in understanding the experiences of stakeholders in AD through their posts online. As a generally novel source of data in the AD literature, online artifacts may shed light on this important topic, while also changing the ways that people live with AD or with the AD of a family member, friend, or client/patient. As was mentioned previously, a dramatic change in the online presence of the average person (within the past decade or more; Internet Live Stats , 2018) might be leading people to engage in Health Co-Inquiry on the Internet, since themes in our analyses do indicate at least some efforts by AD stakeholders to collaborate with others online (e.g., seeking and giving information; searching for and providing resources; sharing emotional supports). With that co-inquiry may come improved education and support for the person with AD, her or his caregivers, and health and mental health providers.

The results of our study indicate that persons with Alzheimer's disease and related dementias post online, although much less than stakeholders without dementia, such as caregivers. Nevertheless, we do see evidence of Health Co-Inquiry among the posts of the former, as in their expressions of fear that their memories are failing and requests for advice about diagnosis and treatments. These types of posts have at their essence: (1) personactivation, and (2) information-seeking. Those are essential parts of Health Co-Inquiry, whereby a person with a health condition is motivated toward improving outcomes and seeks to do so through interactions with other stakeholders (Seifert et al., 2019).

In general, caregivers seemed to be the most frequent users of online forums and blogs about AD. Prominent themes among their posts included seeking and providing information and support, venting emotions, telling one's story, and using posting as a coping tool. The richness of caregiver narratives includes many indicators of Health Co-Inquiry, such as regard for the persons for whom they care, activation toward improving care for the $\mathrm{PwD}$, information-seeking, and reaching out for emotional/other supports. So, many caregivers are "collaborating online" in order to improve outcomes in their own situations.

Presence of other AD stakeholders online is additional evidence for Health Co-Inquiry on the Internet (Seifert et al., 2019). As Table 2 indicates, health and mental health providers, researchers, non-profit organizations, and government agencies are among the other AD stakeholders on the Internet. Their presence makes Health CoInquiry online more likely, because persons with $A D$ or related conditions and their caregivers can access other stakeholder groups via online communications in order to collaborate. As to whether online posting behaviours are different for stakeholders in $A D$ and those in other health conditions, we believe that there may be some similarities across conditions, such as the high frequency of the name of the condition in posts (Seifert et al., 2019). However, it appears to us that there may be a significant difference in AD (and similar conditions that might impair one's cognitive function), which may lead persons with the condition to post less than persons without cognitive impairment. For example, a person with chronic migraine might be more likely to seek support online than a person with $A D$, although this has yet to be evaluated.

Overall, this study provides an important first step toward understanding the use of the Internet by AD stakeholders. It indicates that they are present online and in ways that may foster cooperative inquiry toward improved daily outcomes. Those things are at the very heart of Health Co-Inquiry (Seifert \& Seifert, 2017).

\section{Comparing Methods of Analysis}

Overall, we believe that quantitized data from the robot program are consistent with themes we identified in the qualitative evaluation. Linking qualitative themes with quantitized (web-crawl) data, there are numerous high- 
frequency keywords that relate to the patient-caregiver relationship_especially family relationships-and we noted in our thematic, qualitative work that this happens frequently as caregivers tell their stories. Additional highcount terms regard diagnosis and treatments-things about which persons with AD and caregivers seem to seek and share information. Less populous terms from the web crawl do give us some pause for thought with respect to our inductive, thematic analyses. In the inductive evaluation, we identified that venting emotions is a theme among narratives, especially for caregivers (as was mentioned above). However, terms related to emotions seem less frequent in the web crawl than we might have expected, given the saturation in our qualitative evaluations. Frequencies for negative valence emotional words are: anger (17), things that are "bad" (16), being "angry" (12), frustration ("frustrate" 10), and depression (6). Looking back through the narratives, we see a potential shortcoming of the Bifurcated Method in the current study. It is that negative emotions can be expressed in many different ways, and we might not have identified enough dictionary terms for the web-crawler to adequately capture the true saturation of negative emotions among online posts related to $A D$, which would have led the robot program to show us less emotionality in posts than we had perceived in our inductive, thematic analyses. In addition, because of the many ways that a person might express emotion, even adding more dictionary terms might not solve the problem. As an example, if someone were to write, "I haven't had a good day in a long time," we might be able to identify an expression of negative valence emotion in our inductive analyses, depending on the context, but the web-crawler would not tag the text as such, because none of the corresponding dictionary terms are present.

Alternatively, the web-crawler did reveal something interesting about religiosity/spirituality, i.e., despite our failure to identify it as a theme in our inductive analysis. There are people who mentioned related words in their posts. Frequencies of terms like "pray" (12), "church" (12), and "minister" (11) indicate that there were a few people who mentioned things related to religion. The benefit of the Bifurcated Method, here, is to see an aspect of the data that was not apparent in the inductive, thematic analysis but was by way of the web-crawler.

\section{Limitations}

Limitations of the current study may include the methods by which we found URLs, composed dictionaries of search terms, and vetted both of the aforementioned lists. Moreover, the fact that some URLs block robot programs from crawling them is a disadvantage; as Table 2 indicates, there are some differences in the frequencies of types of sites between the list of crawlable URLS and uncrawlable ones. Finally, our qualitative analyses are most likely subject to personal biases.

In order to guard against researcher bias, we worked independently during the initial qualitative portion of our analyses and (only later) met to compare our notes about themes among the URLs. In addition, we added rigour to our method through its bifurcation, using the web-crawl data to cross-check themes from our qualitative analyses and following the protocols from Seifert et al. (2019). It is our hope that these methodological safeguards have helped to protect the integrity of our work. We note, however, that semantic context is not part of the webcrawling program's functionality; thus, there are limits to what can be gleaned from the comparison between our themes and the crawler's counts. The cross-checking function will have to be further developed in order to be fully advantageous for improving rigour in the Bifurcated Method.

About quantitization of qualitative data, it is admittedly controversial and may be a limitation of our approach (Greene, 2007; Maxwell, 2010; Sandelowski et al., 2009); we share some of the trepidation expressed by Sandelowski et al. (2009). However, we believe that rigour in qualitative analyses can be fortified through quantitization (see Greene, 2007). Furthermore, we agree with Maxwell (2010) that quantitizing may help one to evaluate internal generalisability in qualitative data. Nevertheless, generalisability is a lingering question that remains to be further investigated via a replication study.

\section{Author Disclosure}

All authors have agreed to the preparation of this manuscript and its submission for publication. It has not been submitted to any other journal for consideration for publication.

The authors declare that they have no conflicts of interest related to the current study. 
The Institutional Review Board for our institution reviewed and approved our research protocol; it is also in compliance with the Nuremberg Code and the 1964 Declaration of Helsinki and all its addenda. The study involves archival, publicly available information. Thus, in accordance with US regulations for protection of human subjects, there was no contact with subjects and no consent was sought. We have, however, put in place numerous safeguards. Please, see the "Techniques for Safeguarding Subjects" section.

This study was supported by National Science Foundation CC-NIE Award Number \#1541342 to our institution for technological development of research.

\section{Acknowledgement}

Many thanks to Associate Editors Daneback and Dedkova for their essential advice and guidance and two anonymous Reviewers for their valuable comments and assistance.

\section{References}

Ahola Kohut, S., LeBlanc, C., O'Leary, K., McPherson, A. C., McCarthy, E., Nguyen, C., \& Stinson, J. (2017). The internet as a source of support for youth with chronic conditions: A qualitative study. Child: Care, Health and Development, 44(2), 212-220. https://doi.org/10.1111/cch.12535

Allen, C., Vassilev, I., Lin, S. X., Culliford, D., \& Rogers, A. (2019). The contribution of internet use in personal networks of support for long-term condition self-management. Chronic IIIness, 15(3), 220-235.

https://doi.org/10.1177/1742395318759588

Alzheimer's Research UK. (2018). Dementia statistics hub. https://www.dementiastatistics.org/

Bassuk, S. S., Glass, T. A., \& Berkman, L. F. (1999). Social disengagement and incident cognitive decline in community-dwelling elderly persons. Annals of Internal Medicine, 131(3), 165-173. https://doi.org/10.7326/00034819-131-3-199908030-00002

Cohen, S., \& Wills, T. A. (1985). Stress, social support, and the buffering hypothesis. Psychological Bulletin, 98(2), 310-357. https://doi.org/10.1037/0033-2909.98.2.310

Corbin, J., \& Strauss, A. (1985). Managing chronic illness at home: Three lines of work. Qualitative Sociology, 8(3), 224-247. https://doi.org/10.1007/BF00989485

Coulson, N. S., \& Greenwood, N. (2011). Families affected by childhood cancer: An analysis of the provision of social support within online support groups. Child: Care, Health and Development, 38(6), 870-877.

https://doi.org/10.1111/j.1365-2214.2011.01316.x

Czaja, S. J., Boot, W. R., Charness, N., Rogers, W. A., \& Sharit, J. (2018). Improving social support for older adults through technology: Findings from the PRISM randomized controlled trial. The Gerontologist, 58(3), 467-477. https://doi.org/10.1093/geront/gnw249

Dam, A. E. H., van Boxtel, M. P. J., Rozendaal, N., Verhey, F. R. J., \& de Vugt, M. E. (2017). Development and feasbility of Inlife: A pilot study of an online social support intervention for informal caregivers of people with dementia. PLOS ONE,12(9), Article e0183386. https://doi.org/10.1371/journal.pone.0183386

Frerichs, L., Lich, K. H., Dave, G., \& Corbie-Smith, G. (2016). Integrating systems science and community-based participatory action research to achieve health equity. American Journal of Public Health, 106(2), 215-222.

https://doi.org/10.2105/AJPH.2015.302944

Goode, W. J. (1960). A theory of role strain. American Sociological Review, 25(4), 483-496.

https://doi.org/10.2307/2092933 
Greene, J. (2007). Mixed methods in social inquiry. Jossey-Bass.

Han, A., Radel, J., McDowd, J. M., \& Sabata, D. (2016). Perspectives of people with dementia about meaningful activities: A synthesis. American Journal of Alzheimer's Disease \& Other Dementias, 31(2), 115-123. https://doi.org/10.1177/1533317515598857

Hatch, J. A. (2002). Doing qualitative research in education settings. State University of New York Press.

Holt-Lunstad, J., Robles, T. F., \& Sbarra, D. A. (2017). Advancing social connections as a public health priority in the United States. American Psychologist, 72(6), 517-530. https://doi.org/10.1037/amp0000103

Internet Live Stats. (2018). Internet users. http://www.internetlivestats.com/internet-users/

Kenigsberg, P.-A., Aquino, J.-P., Bérard, A., Gzil, F., Andrieu, S., Banerjee, S., Brémond, F., Buée, L., Cohen-Mansfield, J., Mangialasche, F., Platel, H., Salmon, E., \& Robert, P. (2016). Dementia beyond 2025: Knowledge and uncertainties. Dementia, 15(1), 6-21. https://doi.org/10.1177/1471301215574785

Kim, E., Hou, J., Han, J. Y., \& Himelboim, I. (2016). Predicting retweeting behavior on breast cancer social networks: Network and content characteristics. Journal of Health Communication, 21(4), 479-486.

https://doi.org/10.1080/10810730.2015.1103326

Krueger, K. R., Wilson, R. S., Kamenetsky, J. M., Barnes, L. L., Bienias, J. L., \& Bennett, D. A. (2009). Social engagement and cognitive function in old age. Experimental Aging Research, 35(1), 45-60.

https://doi.org/10.1080/03610730802545028

LaMonica, H. M., English, A., Hickie, I. B., Ip, J., Ireland, C., West, S., Shaw, T., Mowszowski, L., Glozier, N., Duffy, S., Gibson, A. A., \& Naismith, S. L. (2017). Examining Internet and eHealth practices and preferences: Survey study of Australian older adults with subjective memory complaints, mild cognitive impairment, or dementia. Journal of Medical Internet Research, 19(10), Article e358. https://doi.org/10.2196/jmir.7981

Lozano, R., Naghavi, M., Foreman, K., Lim, S., Shibuya, K., Aboyans, V., Abraham, J., Adair, T., Agarwal, R., Ahn, S. Y., Alvarado, M., Anderson, H. R., Anderson, L. M., Andrews, K. G., Atkinson, C., Baddour, L. M., Barker-Collo, S., Bartels, D. H., Bell, M. L., ... Murray, C. J. L. (2012). Global and regional mortality from 235 causes of death for 20 age groups in 1990 and 2010. Lancet, 380(9859), 2095-2128. https://doi.org/10.1016/s0140-6736(12)61728-0

Lum, T. Y., \& Lightfoot, E. (2005). The effects of volunteering on the physical and mental health of older people. Research on Aging, 27(1), 31-55. https://doi.org/10.1177/0164027504271349

Luoh, M.-C., \& Herzog, A. R. (2002). Individual consequences of volunteer and paid work in old age: Health and mortality. Journal of Health and Social Behavior, 43(4), 490-509. https://doi.org/10.2307/3090239

Marks, S. R. (1977). Multiple roles and role strain: Some notes on human energy, time and commitment. American Sociological Review, 42(6), 921-936. https://doi.org/10.2307/2094577

Maxwell, J. A. (2010). Using numbers in qualitative research. Qualitative Inquiry, 16(6), 475-482.

https://doi.org/10.1177/1077800410364740

Ngwenya, N. B., \& Mills, S. (2014). The use of weblogs within palliative care: A systematic literature review. Health Informatics Journal, 20(1), 13-21. https://doi.org/10.1177/1460458213475894

Office for Human Research Protections. (2018). Regulations. https://www.hhs.gov/ohrp/regulations-andpolicy/regulations/index.html 
Oshio, T., \& Kan, M. (2016). How do social activities mitigate informal caregivers' psychological distress? Evidence from a nine-year panel survey in Japan. Health and Quality of Life Outcomes, 14, Article 117.

https://doi.org/10.1186/s12955-016-0521-8

Paulus, T. M., \& Varga, M. A. (2015). "Please know that you are not alone with your pain": Responses to newcomer posts in an online grief support forum. Death Studies, 39(10), 633-640.

https://doi.org/10.1080/07481187.2015.1047060

Pietromonaco, P. R., \& Collins, N. L. (2017). Interpersonal mechanisms linking close relationships to health. American Psychologist, 72(6), 531-542. https://doi.org/10.1037/amp0000129

Prince, M., Prina, M., \& Guerchet, M. (2013). World Alzheimer Report 2013: An analysis of long-term care for dementia. https://www.alz.co.uk/research/world-report-2013

Rogers, A., Vassilev, I., Sanders, C., Kirk, S., Chew-Graham, C., Kennedy, A., Protheroe, J., Bower, P., Blickem, C., Reeves, D., Kapadia, F., Brooks, H., Fullwood, C., \& Richardson, G. (2011). Social networks, work and work-based resources for the management of long-term conditions: A framework and study protocol for developing self-care support. Implementation Science, 6, Article 56. https://doi.org/10.1186/1748-5908-6-56

Rubenstein, E. L. (2015). "They are always there for me": The convergence of social support and information in an online breast cancer community. Journal of the Association for Information Science and Technology, 66(7), 14181430. https://doi.org/10.1002/asi.23263

Sandelowski, M., Voils, C. I., \& Knafl, G. (2009). On quantitizing. Journal of Mixed Methods Research, 3(3), 208-222. https://doi.org/10.1177/1558689809334210

Scharett, E., Madathi, K. C., Lopes, S., Rogers, H., Agnisarman, S., Narasimha, S., Ashok, A., \& Dye, C. (2017). An investigation of the information sought by caregivers of Alzheimer's patients on online peer support groups. Cyberpsychology, Behavior, and Social Networking, 20(10), 640-657. https://doi.org/10.1089/cyber.2017.0274

Seifert, L. S. (2015, November). Is there room for traditional science and action research in aging studies? [Poster presentation]. The Fifth Annual Conference of the Society for Aging \& Society, Washington, DC, USA.

Seifert, L. S., \& Seifert, C. A. (2017). Multi-method Health Co-Inquiry: A case illustration for persons with chronic illness, caregivers, providers, and researchers. Current Psychology, 38(5), 1368-1381.

https://doi.org/10.1007/s12144-017-9676-7

Seifert, L. S., Kaelber, K., Flaherty, K., \& Kromer-Edwards, C. (2019). Using online resources in Health Co-Inquiry: A Bifurcated Method for analyzing stakeholder narratives. Current Psychology, 38(6), 1772-1788.

https://doi.org/10.1007/s12144-019-00474-9

Stanhope, V., \& Henwood, B. F. (2014). Activating people to address their health care needs: Learning from people with lived experience of chronic illness. Community Mental Health Journal, 50(6), 656-663.

https://doi.org/10.1007/s10597-013-9686-3

Stewart, T. (2013). Editorial: Web-based cooperation and collaboration. Behaviour \& Information Technology, 32(6), 517-518. https://doi.org/10.1080/0144929X.2013.805514

Sugihara, Y., Sugisawa, H., Shibata, H., \& Harada, K. (2008). Productive roles, gender, and depressive symptoms: Evidence from a national longitudinal study of late-middle-aged Japanese. The Journals of Gerontology: Series $B$, 63(4), P227-P234. https://doi.org/10.1093/geronb/63.4.P227

Tak, S. H., Zhang, H., Patel, H., \& Hong, S. H. (2015). Computer activities for persons with dementia. The Gerontologist, 55(Suppl. 1), S40-S49. https://doi.org/10.1093/geront/gnv003 
van de Glind, E. M. M., van Enst, W. A., van Munster, B. C., Olde Rikkert, M. G. M., Scheltens, P., Scholten, R. J. P. M., \& Hooft, L. (2013). Pharmacological treatment of dementia: A scoping review of systematic reviews. Dementia and Geriatric Cognitive Disorders, 36(3-4), 211-228. https://doi.org/10.1159/000353892

Vassilev, I., Rogers, A., Sanders, C., Kennedy, A., Blickem, C., Proheroe, J., Bower, P., Kirk, S., Chew-Graham, C., \& Morris, R. (2011). Social networks, social capital, and chronic illness self-management: A realist review. Chronic IIIness, 7(1), 60-86. https://doi.org/10.1177/1742395310383338

Whitlatch, C. J., \& Orsulic-Jeras, S. (2018). Meeting the informational, educational, and psychosocial support needs of person's living with dementia and their family caregivers. The Gerontologist, 58(Suppl. 1), S58-S73. https://doi.org/10.1093/geront/gnx162

Williams, K. N., \& Kemper, S. (2010). Interventions to reduce cognitive decline in aging. Journal of Psychosocial Nursing and Mental Health Services, 48(5), 42-51. https://doi.org/10.3928/02793695-20100331-03

Woodbridge, R., Sullivan, M. P., Harding, E., Crutch, S., Gilhooly, K. J., Gilhooly, M. L. M., Mclntyre, A., \& Wilson, L. (2018). Use of the physical environment to support everyday activities for people with dementia: A systematic review. Dementia, 17(5), 533-572. https://doi.org/10.1177/1471301216648670

Xu, J., Murphy, S. L., Kochanek, K. D., \& Bastian, B. A. (2016). Deaths: Final data for 2013 (National Vital Statistics Reports, Vol. 64, No. 2). National Center for Health Statistics.

https://www.cdc.gov/nchs/data/nvsr/nvsr64/nvsr64_02.pdf

\section{Appendix A: List of Vetted URLs for the AD Web Crawl}

http://ehealthforum.com/health/alzheimers.html

http://forums.moneysavingexpert.com/showthread.php?t=5237647

http://mikegamble.websitetoolbox.com/post/alzheimersdementia-chat-room-2285763

http://thealzheimerspouse.com/vanillaforum/

http://www.abc-directory.com/site/990026

http://www.alzforum.org/

http://www.curezone.org/forums/f.asp?f=21

http://www.healingwell.com/community/?f=8

http://www.healthboards.com/boards/alzheimers-disease-dementia/

http://www.helpforalzheimersfamilies.com/alzheimers-care-training/live-chat-reflections/

http://www.medhelp.org/forums/Alzheimers-Disease/show/379

http://www.nhs.uk/Conditions/Alzheimers-disease/Pages/community.aspx

http://www.psychforums.com/alzheimer/

http://www.topix.com/forum/health/alzheimers-disease

https://ehealthforum.com/health/alzheimers.html

https://forum.alzheimers.org.uk/

https://forums.moneysavingexpert.com/showthread.php?t=5237647

https://patient.info/forums/discuss/browse/alzheimer-s-disease-67

https://www.alzconnected.org/discussion.aspx

https://www.alzforum.org/

https://www.alzu.org/forum/list.php?6

https://www.curezone.org/forums/f.asp?f=21

https://www.dementiacarecentral.com/forum/

https://www.dementiaforum.org/discuss/forum/

https://www.diseasemaps.org/alzheimer-disease/forum/

https://www.healingwell.com/community/?f=8 
https://www.healthboards.com/boards/alzheimers-disease-dementia/

https://www.healthboards.com/boards/epilepsy/

https://www.medhelp.org/forums/Alzheimers-Disease/show/379

https://www.nhs.uk/Conditions/Alzheimers-disease/Pages/community.aspx

https://www.patientslikeme.com/conditions/114-alzheimer-s-disease

https://www.patientslikeme.com/patients

https://www.psychforums.com/alzheimer/

https://www.theguardian.com/lifeandstyle/2011/nov/21/picks-disease-early-onset-dementia

\section{Appendix B: Condition-Specific Dictionary for Alzheimer's Disease*}

Alzheimer //vascular damage //temporal cortex //amyloid precursor protein //synaptopathy //central cholinergic //peripheral cholinergic //cholinergic neuron //semantic dementia //glycogen synthase //brain aging //DYRK1A kinase //DYRK1A //neuron cytoskeleton //amyloid-beta aggregation //A //b-amyloid oligomer toxicity //receptormediated $\beta$-amyloid // $\beta$-amyloid oligomer //mutant $\beta$-amyloid fibrils //automatic memory processing //automatic processing //onset //neuropsychological//dementia //disease //neurofibrillary tangle //NFT //cognitive deficit //impairment//beta-secretase //BACE1//gene discovery //novel loci //TARDBP mutation //TARDBP //TDP-43 //amyloid- $\beta$ //neurovascular defect //hyperphosphorylation of TAU //neurofibrillary //TAU triage //frontotemporal dementia //metal theory //cerebrospinal fluid biomarker //neurodegeneration //vascular brain injury //prevention //assessment //treatment //psychosocial //diffusion tensor //diffusion tensor imaging //biomarker //diagnosis //brain structural imaging //cognitive impairment //white matter //gray matter //neuronal dysfunction //18F-AV-45 //florbetapir //Amyvid //18 $\mathrm{F} / /$ Pittstburgh compound B //position emission //dietary factor //LEARN model //latent early-life associated regulation //intracellular amyloid $\beta$-protein //apomorphine //A $\beta 42$ aggregation //prefrontal cortex //reversal learning impairment //OFBAPP //PrPc //a-secretase //metalloproteinase-mediated //metalloproteinase//metalloprotease //app a-secretase cleavage //B-peptide-mediated //neuronal death //gene regulation //BACE1 //CREB //Fe65 //APBB1 //YY1//y-secretase complex assembly //pro-inflammatory cytokine //modulate glial expression //apolipoprotein E protein //apolipoprotein //lipoprotein receptor //tau-induced neurodegeneration //tauopathy //tauopathies //neuropsychiatric symptom //vascular dementia //fatty aspirin //amyloid- $\beta$ oligomer //human body fluid //early diagnosis //therapy monitoring //visual impairment //clinical pathophysiological //mitochondrial hapgroups //18F-FDG-PET imaging //artificial neural network //milestone //difficulties //difficulty //memory processing //age of onset //neuropsychological feature //significant neuronal loss //toxic effect //spatial pattern //late-onset sporadic Alzheimer's disease //FAD //familial //altered state //brain function //vegetative state //mild cognitive impairment //mitochondrial pathology //calmodulin //Transgenic Model //COX-2 inhibitor //copper studies //copper chelator //anti-Alzheimer //behavioral disturbance //immunotherapy //cerebral amyloid angiopathy //antidepressant //cystatin //cysteine //neurogeneration //acetylcholinesterase-inhibitory potential //acetylcholinesterase //quercetin //AZD-103 //NSAID //symptom //sign //outlook //memory test //blood test //genetic test //brain scan //improving communication //memory aid //losing things //non-recognition //hallucination //restless //agitation //sexual behavior //emotional support //loss //despair //guilt //nursing home //financial capacity //psychological treatment //drug trial //drug //plaque //tau //living brain //new technology //genetic//vascular disease //memory loss //insulin resistance //inflammation //cognitive reserve //drug treatment //gene discovery //neurovascular defect //hyperphosphorylation of TAU //neurofibrillary degeneration //TAU triage //frontotemporal dementia //metal theory //vascular brain injury //risk factor //risk //preventative //hypertension //medicine //brain food //nutrition //body upkeep //physical exercise //cognitive engagement //social network //stage //early onset //late onset

*Due to the length of the list, we have used // to separate terms in order to conserve space.

\section{Appendix C: Global Dictionary of General Terms for All Health Conditions*}

past //present //current //future //mother //wife //husband //spouse //father //parent //child //daughter //son //sister //brother //sibling //grandparent //grandmother //grandfather //friend //peer //peer support //support group //ache //pain //hurts //sick //sickness //ill //illness //injury //accident //sore //symptom //impairment //trauma //onset //treatment //regimen //regime //drug //medicine //prescription //surgery 
//procedure //medical //nurse //doctor //clinician //nurse practitioner //nurse aid //physician's assistant //counselor //social worker //home health aid //health aid //caregiver //carer //hospital //clinic //doctor's office //stability //remission //co- morbid //comorbid //rehabilitation //disability //stress //coping //cope //bad //anger //angry //upset //depressed //depression //shock //frustrate //confuse //irritate //aggravate //irk //sad //upset //joy //relief //happy //good //empower //victory //best //worst //good //bad //walk //drive //exercise //travel //vacation //leisure //work //employ //boss //manage //stigma //faith //pray //church //religion //pastor //priest //minister //parishioner //nun //worship //Bible //Koran //Torah //mindfulness //meditate

*provided by Seifert et al. (2019) 


\section{Correspondence to:}

Lauren S. Seifert

Malone University

2600 Cleveland Ave., NW

Canton

Ohio 44709

Email: LSEIFERT(at)malone.edu

Editorial record: First submission received on February 22, 2019. Revisions received on January 11, 2020, January 15 , 2020, May 6, 2020 and May 27, 2020. Accepted for publication on June 1, 2020.

Editor in charge: Kristian Daneback

\section{About Authors}

Lauren S. Seifert, Ph.D. is a cognitive-experimental psychologist with expertise in cognition, neuroscience, and research methods. She has over thirty years of experience conducting research with elder populations, especially in applied settings with those who have neurodegenerative disorders. She is a Professor of Psychology at Malone University in Canton, Ohio, USA.

Kara Kaelber, Ph.D. is a doctoral-level licensed professional clinical counselor with expertise related to the diagnosis and treatment of mental and emotional disorders. She directs a number of graduate programs at Malone University, including one in clinical counseling. In addition, she oversees the undergraduate psychology program.

Kathleen Flaherty, Ph.D. is a doctoral-level nursing scholar with expertise related to adulthood and geriatric nursing). She is licensed in a number of areas of practice including acute care. She is also a Trustee of the Neuman Systems Model.

Tyler J. Bowman, B.A., is a graduate of Malone University's Psychology program and is currently seeking to work in human services or a related job. 